\title{
Article
}

\section{Digital Workflow for 3D Design and Additive Manufacturing of a New Miniscrew-Supported Appliance for Orthodontic Tooth Movement}

\author{
Daniele Cantarella $^{1, *}$, Vincenzo Quinzi ${ }^{2}$, , Lorena Karanxha ${ }^{1}$, Paolo Zanata ${ }^{3}$, Gianpaolo Savio ${ }^{4}(\mathbb{D})$ \\ and Massimo Del Fabbro 1,5 (D)
}

1 Department of Biomedical, Surgical and Dental Sciences, University of Milan, Via Commenda 10, 20122 Milan, Italy; lorena.karanxha@unimi.it (L.K.); massimo.delfabbro@unimi.it (M.D.F.)

2 Department of Life, Health \& Environmental Sciences, Postgraduate School of Orthodontics, University of L'Aquila, 67100 L'Aquila, Italy; vincenzo.quinzi@univaq.it

3 Lab Technician, Private Practice, Viale delle Querce 3A, 31033 Castelfranco Veneto, Italy; paolo@odontotecnicacastellana.it

4 Department of Civil, Environmental and Architectural Engineering ICEA, University of Padua, Via Venezia 2, 35100 Padova, Italy; gianpaolo.savio@unipd.it

5 Dental Clinic, IRCCS Orthopedic Institute Galeazzi, Via Riccardo Galeazzi 4, 20161 Milan, Italy

* Correspondence: daniele.cantarella@unimi.it

Citation: Cantarella, D.; Quinzi, V.; Karanxha, L.; Zanata, P.; Savio, G.; Del Fabbro, M. Digital Workflow for 3D Design and Additive Manufacturing of a New Miniscrew-Supported Appliance for Orthodontic Tooth Movement. Appl. Sci. 2021, 11, 928. https://doi.org/10.3390/app11030928

Received: 21 December 2020

Accepted: 18 January 2021

Published: 20 January 2021

Publisher's Note: MDPI stays neutral with regard to jurisdictional claims in published maps and institutional affiliations.

Copyright: (c) 2021 by the authors. Licensee MDPI, Basel, Switzerland. This article is an open access article distributed under the terms and conditions of the Creative Commons Attribution (CC BY) license (https:/ / creativecommons.org/licenses/by/ $4.0 /)$.

\begin{abstract}
The introduction of digital workflows and their combination with miniscrew assisted appliances has opened new and enthusiastic perspectives in modern orthodontics. However, in all digital workflows currently in use for orthodontic tooth movement, the miniscrews are inserted first in the maxillary bone, often by means of a surgical guide, and then the appliance is fabricated and secured over the miniscrews with different fixation mechanisms. By doing so, some adaptation problems can be encountered while securing the appliance over the miniscrews, and the chairside time required can therefore be significant. In the present study, we introduce a digital workflow for the design and fabrication of a new appliance, customized on the individual morphology of maxillary bone by using patient Cone Beam Computed Tomography CBCT, for sagittal and vertical orthodontic tooth movement (DIVA, divergent anchors). Differently from the existing protocols, the appliance is cemented first intraorally, serving as a surgical guide for the subsequent insertion of miniscrews. In this way, the adaptation problems are avoided and the chair-side time is reduced.
\end{abstract}

Keywords: digital orthodontics; miniscrew; tooth anchorage; CAD/CAM; laser melting; DIVA; $3 \mathrm{D}$ printing

\section{Introduction}

In the past twenty years, the discipline of orthodontics has experienced revolutionary changes mainly due to two factors: the utilization of temporary anchorage devices (TADs) and, more recently, the development of digital workflows [1].

Miniscrews are being largely used in everyday orthodontic practice and have become a cornerstone for minimizing anchorage loss [2]. While a variety of miniscrew-supported appliances have been proposed for orthodontic movements in the three planes of space, they all share the same sequence of application: first the miniscrews are inserted, then the appliance is secured over the heads of miniscrews either with fixation screws or other mechanisms [3-5]. Such a workflow may have some disadvantages related to difficulties in fitting the appliance to the already inserted miniscrews, the necessity of a surgical guide for a more accurate miniscrew insertion and increased chair time for the clinician.

On the other hand, technology innovation has impacted orthodontics with digital workflows for planning and fabricating miniscrew-supported appliances. A digital planning process involves the utilization of the intraoral scanning file and the cone beam 
computed tomography $(\mathrm{CBCT})$ imaging of maxillary bone for determining the miniscrew position and designing the appliance structure [6]. Additive manufacturing (AM) is used afterwards for the fabrication of the appliance [7]. Such digital workflow offers several advantages. Firstly, it eliminates the inaccuracies introduced by an analog impression taking or manual fabrication of the appliance [8]. Secondly, it enhances the accuracy of the planning process by allowing a better assessment of the miniscrew insertion sites on the basis of patient's CBCT. In this way, a more appropriate temporary anchorage device (TAD) insertion site in terms of bone thickness can be chosen, and any possible damage to nearby structures during miniscrew insertion can be avoided [6]. Thirdly, the AM technology allows for a complete customization of the appliance for each case [9].

In the present study, we introduce a miniscrew-supported appliance for orthodontic dental movement named DIVA (Divergent Anchors), with a digital workflow for planning and selective laser melting (SLM) for fabrication. The appliance is cemented first in the patient's oral cavity, serving as a surgical guide for the subsequent insertion of miniscrews and can provide anchorage for a variety of tooth movements in the sagittal and vertical planes.

\section{Materials and Methods}

The proposed appliance is made of several additively manufactured parts: a main structure that is anchored to the palatal bone by means of 3 miniscrews inserted in the relative bushings, two rods that extend in a distal direction towards the molars and two molar tubes attached to the molar bands (Figure 1). The molar tubes can slide along the rods, to permit a mesial movement of the molars. Hooks are present on the molar tubes and on the anterior portion of the rods to attach elastic chains that generate the force for mesial molar movement.

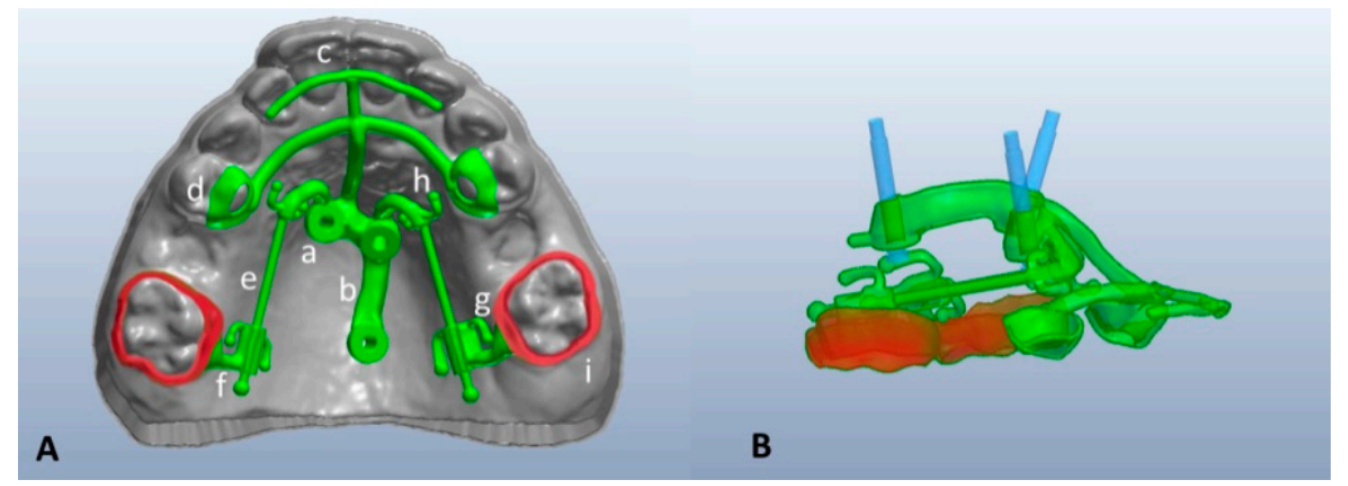

Figure 1. Three-dimensional (3D) design of DIVA (Divergent Anchors) appliance. (A) Occlusal view, showing the parts of the appliance: (a) bushing for the miniscrew (b) connecting bar (c) T-shaped rest on incisors (d) occlusal rest on first premolar (e) rod for guiding mesial movement of molars (f) molar tube attached to molar band (g) hook on molar tube (h) hook on rod (i) conventional prefabricated molar band (in orange). (B) Lateral view, showing that bushings (green) and miniscrews (light blue) are not parallel among them, and slightly diverge in an apical direction. Not parallel miniscrews prevent any vertical movement of the appliance during the clinical use.

To take full advantage of this appliance, an innovative workflow was developed, that is summarized and compared to the conventional approach in Figure 2. Initially, in the proposed workflow, an integrated geometric model is obtained by aligning a virtual model of dental arches and a Cone Beam Computed Tomography (CBCT) image of maxillary bone. Then, the appliance is designed in a computer-aided design (CAD) environment, manufactured by additive manufacturing (AM), laser-welded to prefabricated molar bands, disinfected and finally positioned in the oral cavity. Compared to the conventional approach, in the proposed workflow the appliance presents bushings where miniscrews are 
inserted after the appliance cementation in the oral cavity, to simplify the clinical procedure of appliance delivery.
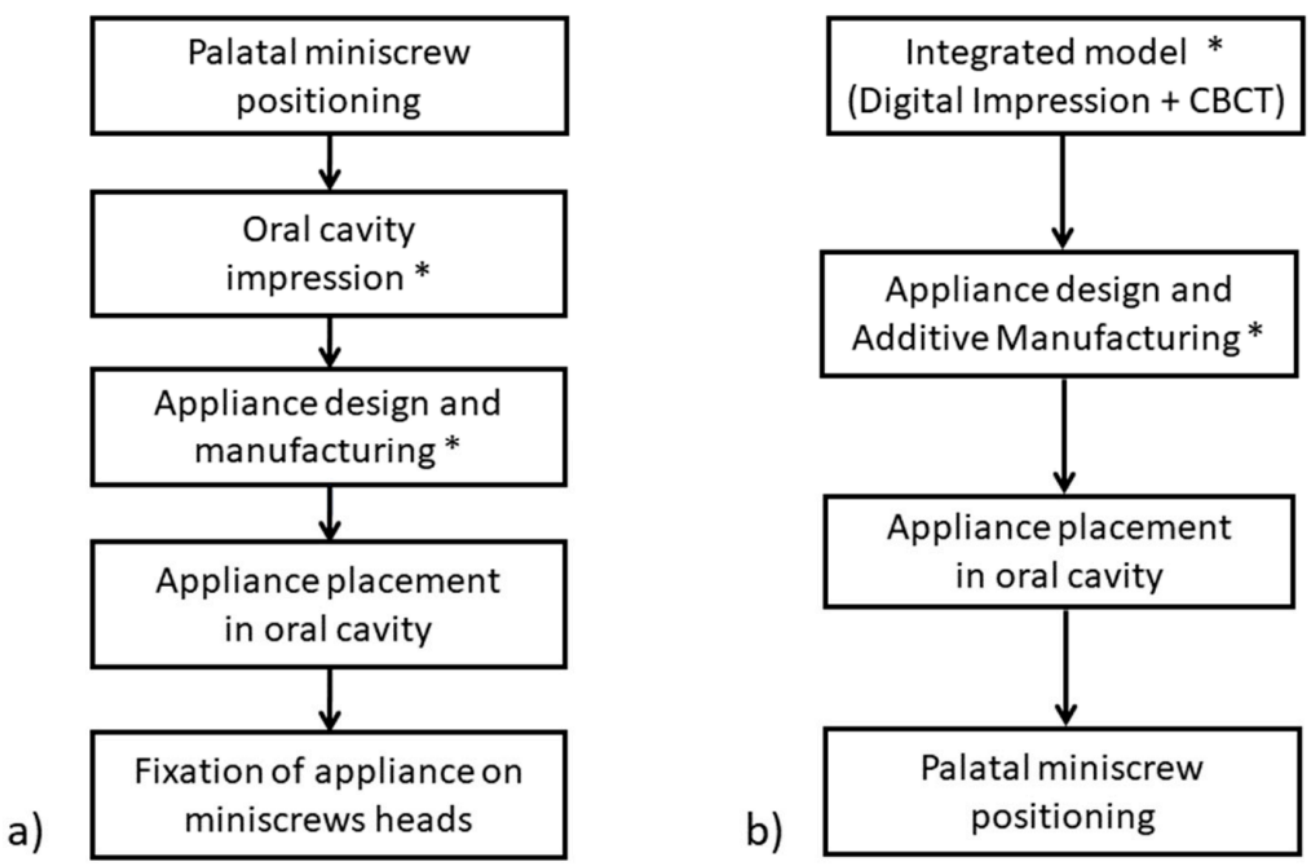

Figure 2. Conventional (a) vs. DIVA (b) workflow. The * symbol indicates a step performed with digital technology.

The impression of patient dental arches was taken with alginate material, stone models were poured and then scanned with Aton scanner (88Dent, Pero, Italy) to obtain a virtual model. Alternatively, the 3D digital model of dental arches can be directly generated with the use of an intra-oral scanner [10]. Then a Cone Beam Computed Tomography (CBCT) exam of the patient maxilla with a Field of View (FOV) of $5 \times 8 \mathrm{~cm}^{2}$ was obtained with a Carestream CS 8100 3D machine (Carestream Dental LLC., Atlanta, GA, USA). The Real Guide software (3Diemme, Figino Serenza, Italy) was used to align the virtual model of the dental arches with the CBCT image through a best-fit automatic algorithm in order to create an integrated model (Figure 3 ) on which the DIVA appliance was subsequently virtually designed.

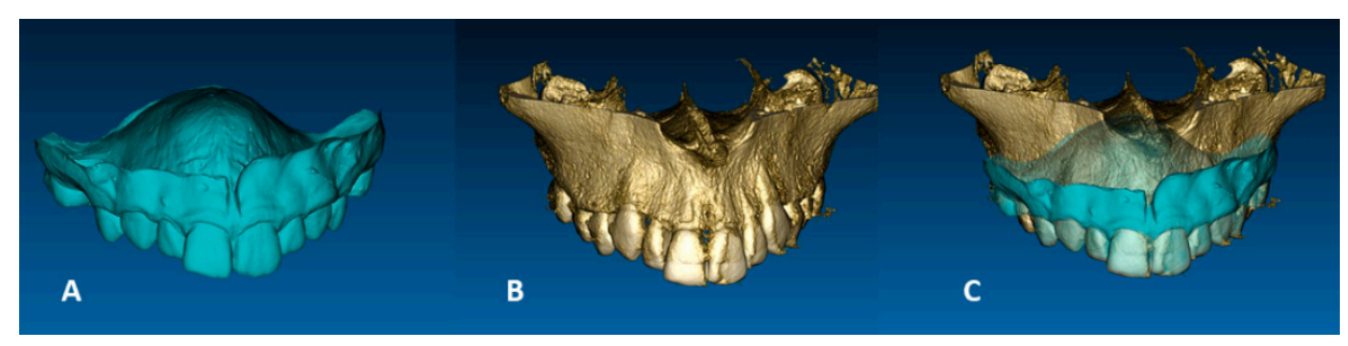

Figure 3. Alignment between virtual model of maxillary dental arch (A) and virtual model of the maxilla cone beam computed tomography $(\mathrm{CBCT})(\mathbf{B})$ to create the integrated model $(\mathbf{C})$.

The appliance was designed in a CAD environment (Rhinoceros 6.0, by Robert McNeel \& Associates, Seattle, WA, USA). A virtual model of a bushing with a cylinder inside, representing the bushing and the miniscrew of the Diva appliance, was generated (Figure 4A,B). The bushing, serving as a guide for the miniscrew, presents height of $4.5 \mathrm{~mm}$, an inner diameter of $1.9 \mathrm{~mm}$, while the external diameter is of $4.9 \mathrm{~mm}$ in the region close to the miniscrew head and of $2.8 \mathrm{~mm}$ towards the opposite end to prevent interference with the palatal mucosa (Figure 4C). 


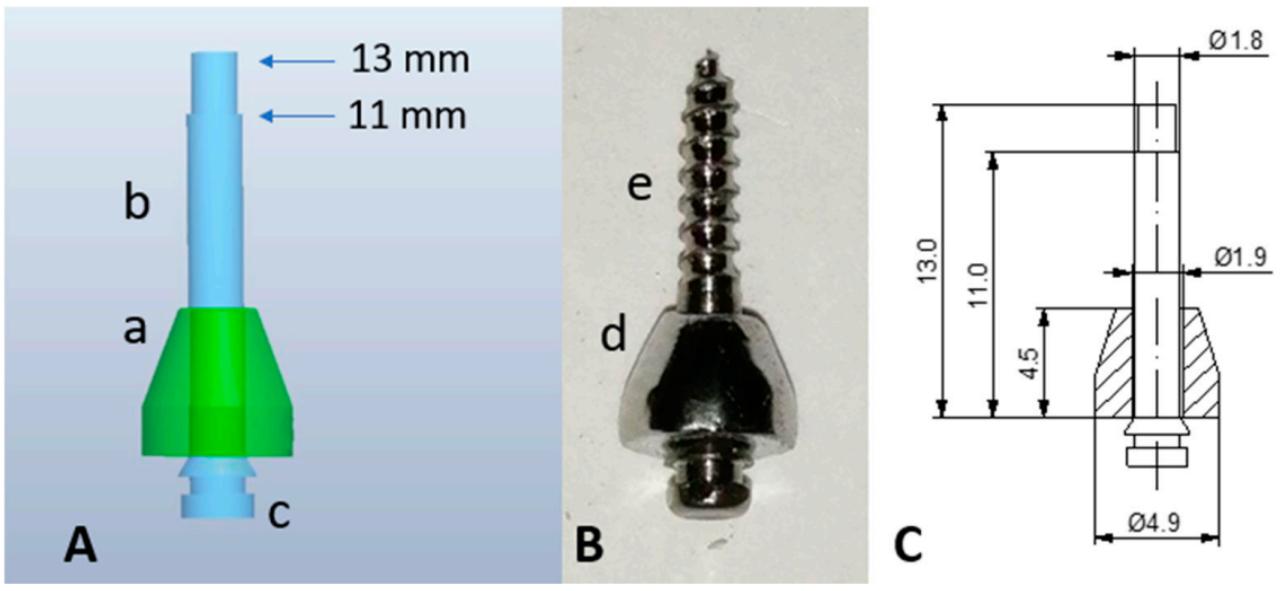

Figure 4. Bushing and miniscrew utilized in the DIVA appliance. (A) Virtual model of the bushing (a) and miniscrew (b). The head of miniscrew is represented by (c). (B) Physical bushing produced with additive manufacturing $(\mathrm{d})$ and conventional prefabricated miniscrew (e). (C) Drawing with dimensions expressed in $\mathrm{mm}$.

The cylinder representing the miniscrew is $13 \mathrm{~mm}$ long and has a diameter of $1.8 \mathrm{~mm}$. Since there are two common lengths for the palatal miniscrews (i.e., $11 \mathrm{~mm}$ and $13 \mathrm{~mm}$ ), to facilitate distinguishing them during virtual planning a shoulder is present at the $11 \mathrm{~mm}, 2 \mathrm{~mm}$ from the most apical end. The other shoulder represents the miniscrew head (Figure 4A,C).

The virtual model of the bushing and miniscrew was then imported into Real Guide software. Three miniscrews with the relative bushings were virtually positioned in the integrated model of the patient maxilla and dental arch. The miniscrew insertion sites were meticulously selected to avoid important anatomical structures like nerves or artery bundles and to maximize the bone thickness at the level of temporary anchorage devices (TADs). Furthermore, the miniscrew bicortical engagement was secured by penetrating the cortical bone layer of the oral cavity vault and nasal floor, to further enforce the TADs primary stability [11], as shown in Figures 5 and 6. Bone thickness at the level of miniscrew insertion sites was measured with the software ruler (Figure 6). These measurements allowed to select the proper length for the miniscrews, that was $13 \mathrm{~mm}$ for the anterior right and left ones, and $11 \mathrm{~mm}$ for the posterior one. Lastly, the three miniscrews were positioned in a non-parallel way, preferably with divergent apical ends, in order to enhance the vertical stability of the appliance during the clinical use (Figures 1 and 5).

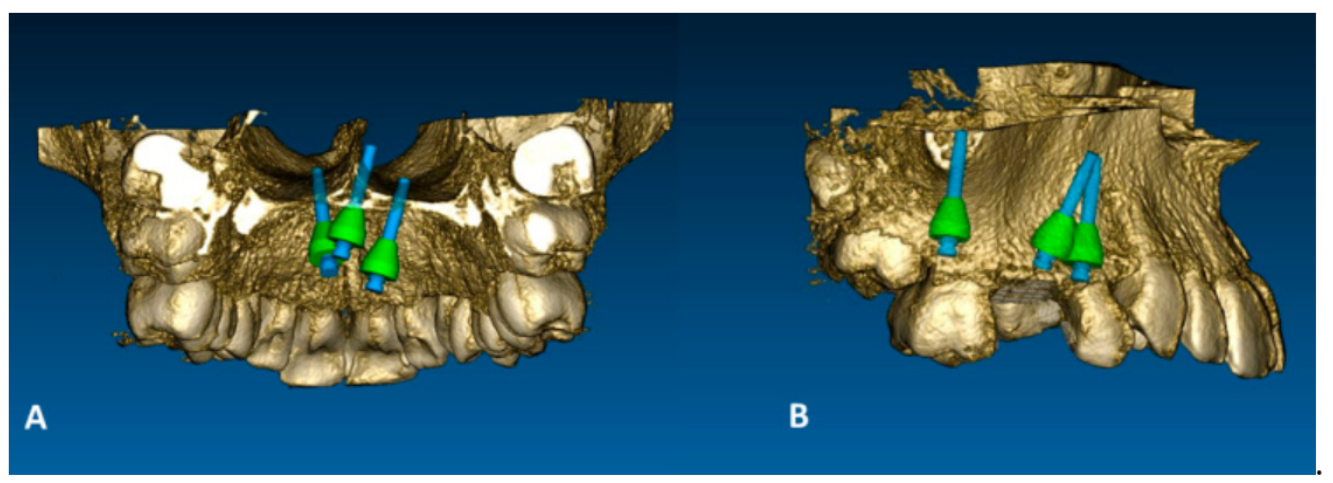

Figure 5. Bushings and miniscrews positioned in the patient CBCT. (A) Posterior view (B) Lateral view. Bushings and miniscrews are positioned in such a way to achieve miniscrew bicortical anchorage (engagement of cortical bone of oral cavity vault and nasal floor). Also, they are not parallel and preferably diverge apically. 


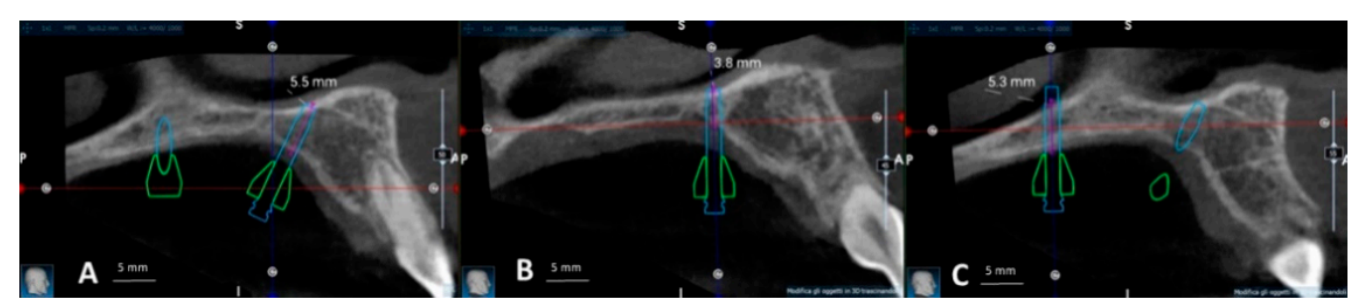

Figure 6. Measurement of bone thickness at miniscrew insertion sites in the patient CBCT with software ruler, and check of miniscrew bicortical engagement. (A) Anterior right miniscrew. (B) Anterior left miniscrew. (C) Posterior miniscrew.

After the placement of the three virtual miniscrews and bushings relative to the patient maxilla CBCT, the Dental Wings software (Dental Wings, Montreal, QC, Canada) was used to design the other parts of the appliance, consisting of: the connecting bars that join the three miniscrew bushings, the T-shaped rest on the lingual surface of the incisors, the rests on the occlusal surface of the premolars, the two longitudinal rods, the molar tubes that are connected to the bands of the maxillary first molars and the hooks (Figure 1). The orientation of the two rods along which the molar tubes slide is designed to guide the sagittal movement of molars, as reported in previous publications $[12,13]$. The rods have a diameter of $1.1 \mathrm{~mm}$, while the molar tubes an inner diameter of $1.2 \mathrm{~mm}$.

Once the virtual design of the appliance was concluded, the fabrication was performed with selective laser melting technology by the Mysint 100 machine (Sisma, Piovene Rocchette, Italy) using the Mediloy S-Co Cobalt-Chromium alloy (Bego, Bremen, Germany). The main processing parameters are summarized in Table 1, while the chemical composition of the Cobalt-Chromium alloy is indicated in Table 2. After AM, heat treatment was performed at $800{ }^{\circ} \mathrm{C}$ for $15 \mathrm{~min}$.

Table 1. Selective Laser Melting processing parameters for Mysint 100 machine utilized in the study.

\begin{tabular}{cc}
\hline Fabrication Parameter & Value \\
\hline Laser power $(\mathrm{W})$ & 90 \\
Scan Speed $(\mathrm{mm} / \mathrm{min})$ & 800 \\
Layer thickness $(\mu \mathrm{m})$ & 20 \\
Hatch spacing $(\mu \mathrm{m})$ & 60 \\
\hline
\end{tabular}

Table 2. Chemical composition of Mediloy S-Co Cobalt Chromium alloy.

\begin{tabular}{cc}
\hline Component & Mass (\%) \\
\hline Co & 63.9 \\
Cr & 24.7 \\
W & 5.4 \\
Mo & 5.0 \\
Si & 1.0 \\
\hline
\end{tabular}

The appliance was manually polished with tungsten burrs and polishing tips with decreasing size of abrasive particles. The molar tubes of the appliance were then laser-welded to conventional prefabricated molar bands. The completed appliance was disinfected with an ethanol and propanol solution (Zeta 7, Zhermack, Badia Polesine, Italy).

The appliance was then cemented in the oral cavity (Figure 7A) and finally the miniscrews were inserted through the appliance bushings into the maxillary bone using the appliance itself as a surgical guide. The premolar occlusal rests are cut immediately after miniscrew insertion, since their only purpose is to help in a precise appliance and miniscrews positioning. An elastic chain was then attached from the hook on molar tubes to the hook on the anterior portion of rods to generate the force for mesial movement of the maxillary molars (Figure 7B). 

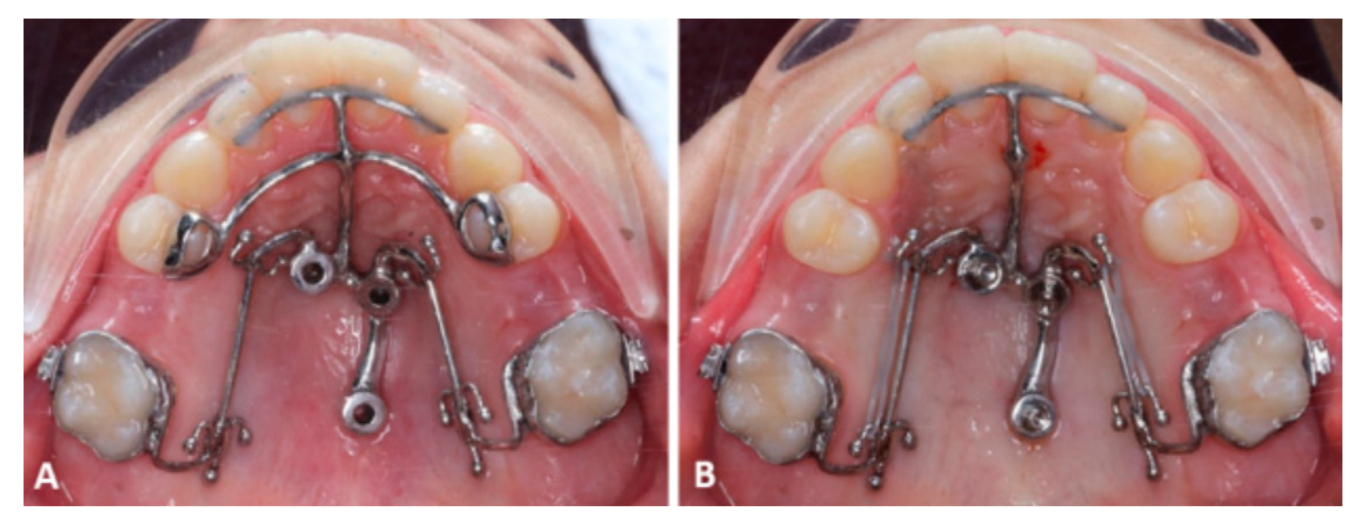

Figure 7. DIVA appliance positioned in the oral cavity. (A) After cementation of the appliance at the level of molar bands, incisal and premolar rests. (B) After insertion of miniscrews through the bushings and placement of elastic chains between the hooks. Occlusal rests on premolars are cut and removed.

\section{Discussion}

Digital orthodontics is gaining more and more acceptance among clinicians in their everyday practice. It started with the intraoral scanning process for the development of virtual models instead of cast ones [14], and is nowadays extending to other processes such as the virtual design of the orthodontic appliances followed by the 3D printing technology [15]. At first the digital workflow was used for simple orthodontic appliances such as trans-palatal arch, lingual arch or Hyrax expander [16]. In the recent years it is being adopted for more complex appliances such as the miniscrew retained ones, which has opened a new and promising perspective in modern orthodontics.

Palatal miniscrew assisted appliances can be divided into two main groups based on their target movement: appliances for the transverse expansion of the maxilla $[17,18]$ and appliances for sagittal and vertical dental movements (mesialization, distalization, intrusion, extrusion). For the latter group, a variety of appliances have been proposed [19-21] but only recently a digital workflow has been incorporated [3-5,22-24]. In all digital workflows currently in use, the miniscrews are inserted first, often with the help of a surgical guide, and the appliance is secured afterwards with a fixation mechanism [3-5].

The methodology for the DIVA appliance of the present study differs from the common workflow, in that the appliance is the first component to be cemented intraorally, and the miniscrews are inserted subsequently with the appliance serving as a surgical guide for their insertion. This workflow avoids the need for a surgical guide for the miniscrews insertion and reduces the chair-time in office by simplifying the clinical procedure.

Cantarella and colleagues previously reported a similar protocol for Maxillary Skeletal Expander (MSE), a particular type of miniscrew assisted rapid palatal expander [25]. Instead, the purpose of the DIVA appliance presented in this study is to generate the sagittal or vertical movement of teeth. In addition, the appliance is fully customized and entirely produced with additive manufacturing (AM) technology, with the exception of molar bands. Although the production of molar bands with AM technology could have further simplified the workflow, conventional prefabricated bands are still very common in the orthodontic clinical practice, because they are made with a more ductile material, that offers a better retention and less accidental debonding. Adhesion of bands produced with AM technology is object of future studies.

Given that the appliance is serving as a surgical guide, its precise positioning in the oral cavity is of crucial importance. For this reason, the occlusal rests on the premolars are included in the initial design of the appliance and serve as a guide for the positioning of the DIVA appliance in the oral cavity. They are immediately cut after the insertion of the miniscrews.

Another important aspect of the DIVA appliance is its vertical stability. In fact, during tooth movement, the appliance could accidentally move apically towards the palatal 
mucosa and create tissue impingement. To avoid such an occurrence, in the phase of design of the appliance, the virtual bushings are not placed parallel, but preferably with a slight apical divergence. Since the bushings are the guide for the miniscrew insertion, miniscrews are also going to be not parallel after their insertion in the maxillary bone. This lack of parallelism will create undercuts between the miniscrews and the main structure of the appliance, which avoids any vertical movement during the orthodontic treatment. Kook et al. [24] have previously introduced the modified C-palatal plate (MCPP), which is a preformed appliance that can be adapted to the individual shape of the patient palate with a plier by the orthodontist. The plate presents 3 holes where miniscrews can then be inserted. However, the plate is preformed and the position of miniscrews cannot be customized. For the DIVA appliance, the position of the miniscrews is decided for each patient, based on the CBCT, offering the previously mentioned advantages in terms of safety and TADs primary stability.

The last aspect to consider is the fabrication of the appliance. The fabrication process of metal appliances has undergone fundamental changes passing from the conventional methods of metal fusion to the powder bed fusion additive manufacturing technologies such as the Electric Beam Melting and the Selective Laser Melting (SLM) [26,27]. They differ from the source of heat they use to melt the metal alloy. Thanks to the layer manufacturing approach, it is possible to fabricate geometrically complex and customized parts which could not be realized with a conventional manufacturing process [28]. For DIVA appliance the SLM was adopted, which exploits a laser source that completely melts the alloy powder, thus obtaining high-density materials [29]. Cobalt-chromium alloy powder, often used in the dental field, was selected because of its excellent mechanical properties, durability and biocompatibility [30].

Although the specific appliance shown in this study has the scope to move the maxillary molars in a mesial direction, a slight change in its design can be used to obtain other dental movements like distalization, intrusion, extrusion or rotation.

\section{Conclusions}

The present study introduces the miniscrew-supported DIVA appliance for orthodontic tooth movement, customized on the morphology of maxillary bone through the patient $\mathrm{CBCT}$, and the complete digital protocol from its virtual design to the additive manufacturing with the selective laser melting technique. This is the first digital protocol in which a customized miniscrew retained appliance for sagittal or vertical orthodontic tooth movement is cemented first and serves as a surgical guide for miniscrews insertion. Furthermore, the concept of not parallel, preferably apically divergent miniscrews is introduced for a better vertical stability of the appliance during the clinical use.

Author Contributions: Conceptualization, D.C., V.Q. and P.Z.; Data curation, D.C., L.K. and G.S.; Formal analysis, D.C. and M.D.F.; Methodology, D.C., P.Z. and G.S.; Project administration, M.D.F.; Resources, D.C., P.Z., G.S. and V.Q.; Software: D.C., P.Z. and G.S.; Supervision: M.D.F.; Validation: D.C., P.Z.; Writing—original draft, D.C. and L.K.; Writing—review \& editing: D.C., L.K., V.Q., P.Z. and G.S. All authors have read and agreed to the published version of the manuscript.

Funding: This research received no external funding.

Institutional Review Board Statement: The study was conducted according to the guidelines of the Declaration of Helsinki, and approved by the Institutional Review Board of the IRCCS Orthopedic Institute Galeazzi (protocol code L2057, No. 75/2019, date 18/06/2019).

Informed Consent Statement: Informed consent was obtained from all subjects involved in this study.

Data Availability Statement: Data are available from the corresponding Author upon request.

Conflicts of Interest: The authors declare no conflict of interest. 


\section{References}

1. Ghafari, J.G. Centennial inventory: The changing face of orthodontics. Am. J. Orthod. Dentofac. Orthop. 2015, 148, 732-739. [CrossRef] [PubMed]

2. Cornelis, M.A.; Scheffler, N.R.; De Clerck, H.J.; Tulloch, J.F.C.; Behets, C.N. Systematic review of the experimental use of temporary skeletal anchorage devices in orthodontics. Am. J. Orthod. Dentofac. Orthop. 2007, 131 (Suppl. S4), 52-58. [CrossRef] [PubMed]

3. Graf, S.; Vasudavan, S.; Wilmes, B. CAD/CAM Metallic Printing of a Skeletally Anchored Upper Molar Distalizer. J. Clin. Orthod. 2020, 54, 140-150. [PubMed]

4. Maino, B.G.; Paoletto, E.; Lombardo, L.; Siciliani, G. A Three-Dimensional Digital Insertion Guide for Palatal Miniscrew Placement. J. Clin. Orthod. 2016, 50, 12-22. [PubMed]

5. De Gabriele, O.; Dallatana, G.; Riva, R.; Vasuvdavan, S.; Wilmes, B. The Easy Driver for Placement of palatal mini-implants and a maxillary expander in a single appointment. J. Clin. Orthod. 2017, 51, 728-737. [PubMed]

6. Graf, S.; Hansa, I. Clinical guidelines to integrate temporary anchorage devices for bone-borne orthodontic appliances in the digital workflow. APOS Trends Orthod. 2019, 9, 182-189. [CrossRef]

7. Graf, S. Direct Printed Metal Devices-The Next Level of Computer-aided Design and Computer-aided Manufacturing Applications in the Orthodontic Care. APOS Trends Orthod. 2017, 7, 253-259. [CrossRef]

8. Chochlidakis, K.M.; Papaspyridakos, P.; Geminiani, A.; Chen, C.J.; Feng, I.J.; Ercoli, C. Digital versus conventional impressions for fixed prosthodontics: A systematic review and meta-analysis. J. Prosthet. Dent. 2016, 116, 184-190.e12. [CrossRef]

9. Vaid, N.R. Up in the Air: Orthodontic technology unplugged! APOS Trends Orthod. 2017, 7, 1-5. [CrossRef]

10. Di Fiore, A.; Meneghello, R.; Graiff, L.; Savio, G.; Vigolo, P.; Monaco, C.; Stellini, E. Full arch digital scanning systems performances for implant-supported fixed dental prostheses: A comparative study of 8 intraoral scanners. J. Prosthodont. Res. 2019, 63, 396-403. [CrossRef]

11. Lee, R.J.; Moon, W.; Hong, C. Effects of monocortical and bicortical mini-implant anchorage on bone-borne palatal expansion using finite element analysis. Am. J. Orthod. Dentofac. Orthop. 2017, 151, 887-897. [CrossRef] [PubMed]

12. Carano, A.; Testa, M. The distal jet for upper molar distalization. J. Clin. Orthod. 1996, 30, 374-380. [PubMed]

13. Wilmes, B.; Drescher, D. A miniscrew system with interchangeable abutments. J. Clin. Orthod. 2008, 42, 574-580. [PubMed]

14. Claus, D.; Radeke, J.; Zint, M.; Vogel, A.B.; Satravaha, Y.; Kilic, F.; Hibst, R.; Lapatki, B.G. Generation of 3D digital models of the dental arches using optical scanning techniques. Semin Orthod. 2018, 24, 416-429. [CrossRef]

15. Tarraf, N.E.; Ali, D.M. Present and the future of digital orthodontics. Semin Orthod. 2018, 24, 376-385. [CrossRef]

16. Graf, S.; Cornelis, M.A.; Hauber Gameiro, G.; Cattaneo, P.M. Computer-aided design and manufacture of hyrax devices: Can we really go digital? Am. J. Orthod. Dentofac. Orthop. 2017, 152, 870-874. [CrossRef]

17. Baik, H.S.; Kang, Y.G.; Choi, Y.J. Miniscrew-assisted rapid palatal expansion: A review of recent reports. J. World Fed. Orthod. 2020, 9, S54-S58. [CrossRef]

18. Carlson, C.; Sung, J.; McComb, R.W.; MacHado, A.W.; Moon, W. Microimplant-assisted rapid palatal expansion appliance to orthopedically correct transverse maxillary deficiency in an adult. Am. J. Orthod. Dentofac. Orthop. 2016, 149, 716-728. [CrossRef]

19. Kinzinger, G.; Wehrbein, H.; Byloff, F.K.; Yildizhan, F.; Diedrich, P. Innovative Verankerungsalternativen zur Molarendistalisation im Oberkiefer-Eine Übersicht. J. Orofac. Orthop. 2005, 66, 397-413. [CrossRef]

20. Karaman, A.I.; Basciftci, F.A.; Polat, O. Unilateral Distal Molar Movement with an Implant-Supported Distal Jet Appliance. Angle Orthod. 2002, 72, 167-174.

21. Wilmes, B.; Drescher, D.; Nienkemper, M. A miniplate system for improved stability of skeletal anchorage. J. Clin. Orthod. 2009, 43, 494-501.

22. Wilmes, B.; Nanda, R.; Nienkemper, M.; Ludwig, B.; Drescher, D. Correction of upper-arch asymmetries using the MesialDistalslider. J. Clin. Orthod. 2013, 47, 648-655. [PubMed]

23. Papadopoulos, M.A. Efficient Distalization of Maxillary Molars with Temporary Anchorage Devices for the Treatment of Class II Malocclusion. Turk. J. Orthod. 2020, 33, 197-201. [CrossRef] [PubMed]

24. Kook, Y.A.; Bayome, M.; Trang, V.T.T.; Kim, H.J.; Park, J.H.; Kim, K.B.; Behrents, R.G.G. Treatment effects of a modified palatal anchorage plate for distalization evaluated with cone-beam computed tomography. Am. J. Orthod. Dentofac. Orthop. 2014, 146, 47-54. [CrossRef] [PubMed]

25. Cantarella, D.; Savio, G.; Grigolato, L.; Zanata, P.; Berveglieri, C.; Giudice, A.L.L.; Isola, G.; Del Fabbro, M.; Moon, W. A new methodology for the digital planning of micro-implant-supported maxillary skeletal expansion. Med. Devices 2020, 13, 93-106. [CrossRef]

26. Luis, E.; Pan, H.M.; Sing, S.L.; Bastola, A.K.; Goh, G.D.; Goh, G.L.; Tan, H.K.K.J.; Bajpai, R.; Song, J.; Yeong, W.Y. Silicone 3D Printing: Process Optimization, Product Biocompatibility, and Reliability of Silicone Meniscus Implants. 3D Print Addit. Manuf. 2019, 6, 319-332. [CrossRef]

27. Yan, Q.; Dong, H.; Su, J.; Han, J.; Song, B.; Wei, Q.; Shi, Y. A Review of 3D Printing Technology for Medical Applications. Engineering 2018, 4, 729-742. [CrossRef]

28. Savio, G.; Rosso, S.; Meneghello, R.; Concheri, G. Geometric modeling of cellular materials for additive manufacturing in biomedical field: A review. Appl. Bionics Biomech. 2018, 2018, 1654782. [CrossRef]

29. Vandenbroucke, B.; Kruth, J.P. Selective laser melting of biocompatible metals for rapid manufacturing of medical parts. Rapid Prototyp. J. 2007, 13, 196-203. [CrossRef]

30. Di Fiore, A.; Savio, G.; Stellini, E.; Vigolo, P.; Monaco, C.; Meneghello, R. Influence of ceramic firing on marginal gap accuracy and metal-ceramic bond strength of 3D-printed Co-Cr frameworks. J. Prosthet. Dent. 2020, 124, 75-80. [CrossRef] 